PEERS Participants should feel safe enough to share their experiences, actions and reactions in open forum, their attendance and participation is never mandated, Participants are never compelled or required to share before they are ready.

\section{A QUALITATIVE EVALUATION OF A 1 YEAR PILOT STUDY OF YOUNG PEOPLE AND PARENTS ACCESSING A MENTAL HEALTH DROP-IN CENTRE IN A PAEDIATRIC HOSPITAL SETTING}

Kate Fifield, Laila Xu, Natalia Rojas, Matteo Catanzano, Sophie Bennett, Charlotte Sanderson, Anna Coughtrey, Ellie Kerry, Holan Liang, Isobel Heyman, Roz Shafran. Great Ormond Street Hospital and University College London

\subsection{6/archdischild-2020-gosh.40}

Background Children and young people with long term physical health conditions (LTC) are known to have higher levels of co-morbid mental health problems than medically healthy children. Evidence-based treatments for mental health problems are effective in children who also have an LTC. A dropin centre in a paediatric hospital, delivering a range of interventions including onward referral, signposting and guided self-help, may be one way to complement existing mental health services as part of a stepped-care approach. The aim of this study was to understand patients' perspectives of the centre.

Methods 128 patients attending a drop-in centre at Great Ormond Street Hospital were invited to participate. Overall, 35 participated in semi-structured interviews (either in person or by phone) exploring their experience of the drop-in centre. Interviews were audio-recorded, transcribed, and analysed using Framework analysis.

Results Overall, participants found the drop-in centre highly acceptable and reported a positive experience. Reasons for this varied, but broadly focused around four areas:(1) Choice in how to access the intervention and feeling empowered afterwards; (2) Having someone to talk to who could provide them with practical support; (3) Integration and parity of physical and mental health care; (4) The intervention being sufficient to meet their needs and quick to access.

Discussion Participants found the intervention feasible and acceptable. A drop-in centre in a paediatric hospital appears to be a positive and valued adjunct to supplement existing mental health services at GOSH.

\section{FHIR-ENABLED HOLOGRAPHIC MODELS FROM IMAGING STUDIES: HOLOREPOSITORY 2020}

${ }^{1}$ Abhinath Kumar, ${ }^{1}$ Immanuel Baskaran, ${ }^{1}$ Carlo Winkelhake, ${ }^{1}$ Daren Alfred, ${ }^{2}$ Owen Arthurs, ${ }^{3}$ Costas Stylianou, ${ }^{3}$ Chris Feltham, ${ }^{3}$ Phillippa Chick, ${ }^{1}$ Dean Mohamedally, ${ }^{4}$ Neil J Sebire, ${ }^{5}$ Sheena Visram. ${ }^{1}$ UCL Department of Computer Sciences; ${ }^{2}$ Great Ormond Street Hospital; ${ }^{3}$ Intel; ${ }^{4}$ GOSH DRIVE

\subsection{6/archdischild-2020-gosh.41}

Introduction Advancements in Augmented Reality technologies allow for the generation of three-dimensional (3D) models of anatomical structures from MRI or CT scans. We present the HoloRepository 2020 Proof of Concept as an open source FHIR-enabled research demonstrator using openly available imaging data.

Method We used the latest Machine Learning (ML) algorithms in the field for organ segmentation with a supporting structure for future ML algorithm revisions. The Cancer Imaging Archive was used for testing and integration, providing over 30 million radiology images for over 70 different anatomical structures. With this, a model training process that will allow hospitals and clinical groups to build out organ segmentation models on their own DICOM sets is anticipated.

Results This new version has 3 main editions: the Cloud HoloRepository 2020 (CH20), Intel NUC optimized HoloRepoistory 2020 (NH20), and HoloRepository 2020 Viewer (H20V). These facilitate new components such as an organ segmentation library, enhanced augmented reality experiences through cameras and multi-monitor displays, and a synthetic medical imaging data platform. CT and MRI DICOM scans of the brain, lungs, chest, abdomen and kidneys are rendered as a $3 \mathrm{D}$ view using a pre-trained model and the latest techniques for organ segmentation. A step-by-step guide on how to carry out the implementation procedure from research to deployment is laid out, enabling easy integration of newly available segmentation algorithms and methods.HoloRegistration enables simplified over-the-body tracking of the holographic views, achieving an Augmented Reality clinical education experience. Conclusion The viewer and Intel NUC application allows for HoloRepository to be run on a local laptop or workstation, providing easier and faster access. Optimisations from this study have reduced generation times from 30 seconds to 3 seconds and cloud hosting costs by $66 \%$. Future research into volumetric measurements and 2D cross-sectional tracking may aid diagnostics and assist navigation during surgery.

\section{AUGMENTED REALITY CLIPBOARD: A SMART, SECURE AND INTERACTIVE ALTERNATIVE FOR PATIENT OBSERVATION CHARTING}

${ }^{1}$ Lilly Sinek, ${ }^{2}$ Neil J Sebire, ${ }^{3}$ Sheena Visram. ${ }^{1}$ UCL Department of Computer Sciences; ${ }^{2}$ GOSH DRIVE; ${ }^{3}$ University College London

\subsection{6/archdischild-2020-gosh.42}

Introduction In a hospital, a patient may traditionally have observations and personal data displayed on a paper clipboard next to their bed. Not only does this raise privacy concerns, as data can be viewed by anyone approaching the bed, but it also needs to be manually updated when there is a change. A handheld mobile Augmented Reality (AR) application allows the clinician to view data as superimposed on a real-life clipboard, fetching and updating this information in real time. The AR application draws the clinician away from the screen and back into the 'real world', human interaction with the patient, providing a seamless, unobtrusive way for them to interact with patient data.

Method As part of a collaboration between GOSH and UCL computer science through the industry exchange network (IXN), we developed a prototype mobile AR application for viewing patient data on an AR clipboard. The application was developed using Unity game engine and the AR foundation package for cross platform development with iOS and Android. The application pulled synthetic patient data for display, in the FHIR standard format, from the FHIR API.

Results The mobile AR application responds to a trigger image on a clipboard and displays patient information in real time, tracking the clipboard in space, so the information appears superimposed onto the object. The application offers the option to search and display patient data by patient ID 